ARTICLE

DOI: $10.1038 / s 41467-017-01367-w$

\title{
Structural basis for high-affinity actin binding revealed by a $\beta$-III-spectrin SCA5 missense mutation
}

\author{
Adam W. Avery', Michael E. Fealey (10 ${ }^{2}$, Fengbin Wang (iD ${ }^{3}$, Albina Orlova ${ }^{3}$, Andrew R. Thompson (i) ${ }^{2}$, \\ David D. Thomas (10) ${ }^{2}$, Thomas S. Hays ${ }^{1}$ \& Edward H. Egelman (1) ${ }^{3}$
}

Spinocerebellar ataxia type 5 (SCA5) is a neurodegenerative disease caused by mutations in the cytoskeletal protein $\beta$-III-spectrin. Previously, a SCA5 mutation resulting in a leucine-toproline substitution (L253P) in the actin-binding domain (ABD) was shown to cause a 1000fold increase in actin-binding affinity. However, the structural basis for this increase is unknown. Here, we report a $6.9 \AA$ cryo-EM structure of F-actin complexed with the L253P $A B D$. This structure, along with co-sedimentation and pulsed-EPR measurements, demonstrates that high-affinity binding caused by the $\mathrm{CH}$-localized mutation is due to opening of the two $\mathrm{CH}$ domains. This enables $\mathrm{CH} 1$ to bind actin aided by an unstructured $\mathrm{N}$-terminal region that becomes $\alpha$-helical upon binding. This helix is required for association with actin as truncation eliminates binding. Collectively, these results shed light on the mechanism by which $\beta$-III-spectrin, and likely similar actin-binding proteins, interact with actin, and how this mechanism can be perturbed to cause disease.

\footnotetext{
${ }^{1}$ Department of Genetics, Cell Biology and Development, University of Minnesota, Minneapolis, MN 55455, USA. ${ }^{2}$ Department of Biochemistry, Molecular Biology and Biophysics, University of Minnesota, Minneapolis, MN 55455, USA. ${ }^{3}$ Department of Biochemistry and Molecular Genetics, University of Virginia, Charlottesville, VA 22908, USA. Thomas S. Hays and Edward H. Egelman jointly supervised this work. Correspondence and requests for materials should be addressed to T.S.H. (email: haysx001@umn.edu)
} 
S pinocerebellar ataxia type 5 (SCA5) is a neurodegenerative disease that stems from autosomal dominant mutations in the cytoskeletal protein $\beta$-III-spectrin ${ }^{1,2}$. SCA5 pathogenesis results from a functional deficit in Purkinje cells, in which the expression of $\beta$-III-spectrin is required for normal cerebellar control of motor coordination ${ }^{3}$. $\beta$-III-spectrin is thought to form a heterotetrameric complex with $\alpha$-II-spectrin, and to cross-link actin filaments to form a cytoskeleton localizing to the shafts and spines of Purkinje cell dendrites. $\beta$-III-spectrin is required for normal dendrite structure ${ }^{4}$ and synaptic transmission ${ }^{5}, 6$. Recently, our group reported that a SCA5 missense mutation, L253P, localized to the $\beta$-III-spectrin N-terminal actin-binding domain (ABD), causes a $\sim 1000$-fold increase in actin-binding affinity $^{7}$. Here, we probe the structural mechanism of this mutation by studying the $\mathrm{ABD}$ with complementary biophysical techniques.

The $\beta$-III-spectrin ABD comprises tandem calponin homology $(\mathrm{CH})$ domains and the L253P mutation is localized to the second subdomain $(\mathrm{CH} 2)$. Very little is known about the structural biology of $\beta$-III-spectrin's $\mathrm{ABD}$, with the closest related atomic model being the isolated $\mathrm{CH} 2$ domain of $\beta$-II-spectrin ${ }^{8}$. Crystal structures of $\mathrm{N}$-terminal ABDs from the spectrin superfamily, including $\alpha$-actinin, dystrophin and utrophin, invariably show that extensive contacts are made between $\mathrm{CH} 1$ and $\mathrm{CH} 2$, suggesting a tendency to exist in a "closed" conformation in the absence of actin ${ }^{9-12}$. A cryo-EM structure of the fimbrin ABD shows that it associates with actin in a closed structural state ${ }^{13}$. In contrast, cryo-EM showed that $\alpha$-actinin associates with actin in an "open" structural state in which only a single $\mathrm{CH}$ domain is bound to the filament and the second domain is structurally disordered on account of it being dissociated from the interacting $\mathrm{CH}$ domain ${ }^{14}$. A similar conclusion was reached for filamin ${ }^{15}$, another member of the spectrin superfamily. Binding studies suggested that the $\mathrm{CH} 1$ domain of $\alpha$-actinin has greater intrinsic affinity for actin in isolation ${ }^{16}$ and this suggested that it was $\mathrm{CH} 1$ bound in the cryo-EM structure. This led to the hypothesis that the $\mathrm{CH} 2$ domain functions to regulate the actin-binding function of $\mathrm{CH} 1$ through steric hindrance when the two domains are associated. Consistent with this, many mutations in the $\mathrm{CH} 2$ domains of both $\alpha$-actinin and filamin impart modest gains in $\mathrm{ABD}$ affinity for $\operatorname{actin}^{17,18}$. Collectively, these studies suggest that the L253P mutation of $\beta$-III-spectrin, which is similarly localized to $\mathrm{CH} 2$, causes high-affinity actin binding by disrupting a regulatory mechanism that shifts the $\mathrm{ABD}$ structural equilibrium from a closed to more open binding-competent state. Here, we report cryo-EM, co-sedimentation, and pulsed electron paramagnetic resonance (EPR) data consistent with such a mechanism.

\section{Results}

Structure of L253P $\beta$-III-spectrin ABD bound to actin. To begin testing our hypothesis, we first performed cryo-EM on the $\beta$-III-spectrin ABD bound to actin filaments. The actin-binding affinity of the wild-type (WT) $\beta$-III-spectrin ABD is low $\left(K_{\mathrm{d}}=75\right.$ $\mu \mathrm{M})$, resulting in poorly decorated actin filaments that were of insufficient quality for analysis (Supplementary Fig. 1a). The L253P ABD yielded high-quality complexes of decorated filaments (Supplementary Fig. 1b, c), enabling a three-dimensional reconstruction (Fig. 1a) of the mutant $\mathrm{ABD}$-actin complex at 6.9
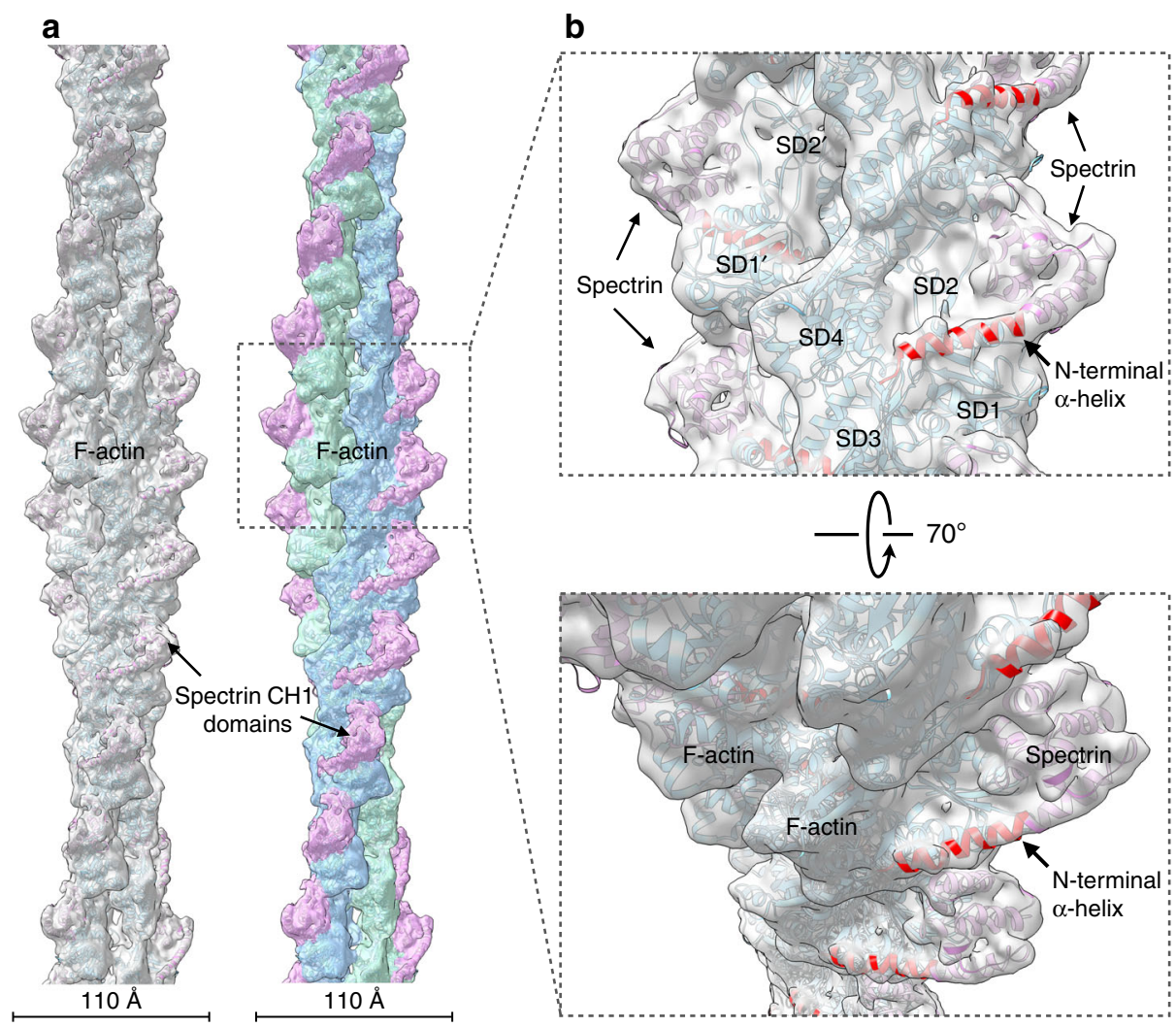

Fig. 1 Cryo-EM map and model of L253P $\beta$-III-spectrin ABD bound to actin. a The map (left, gray transparent surface) has been fit with a model for actin (cyan) and the $\beta$-III-spectrin ABD (magenta). On the right, the surface of the reconstruction has been color coded for the two actin strands (blue and green) and the $\beta$-III-spectrin ABD (magenta). b Close-up view of a showing that the $\mathrm{CH} 1$ domain has an additional $\mathrm{N}$-terminal helix (red) interacting with F-actin. The actin subdomains (SD1, SD2, SD3, and SD4) have been labeled on one actin subunit, while SD1' and SD2' are labeled on a different subunit 
a

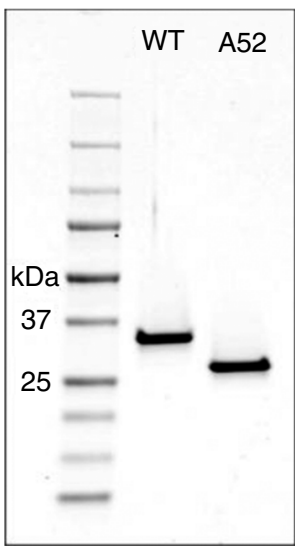

b

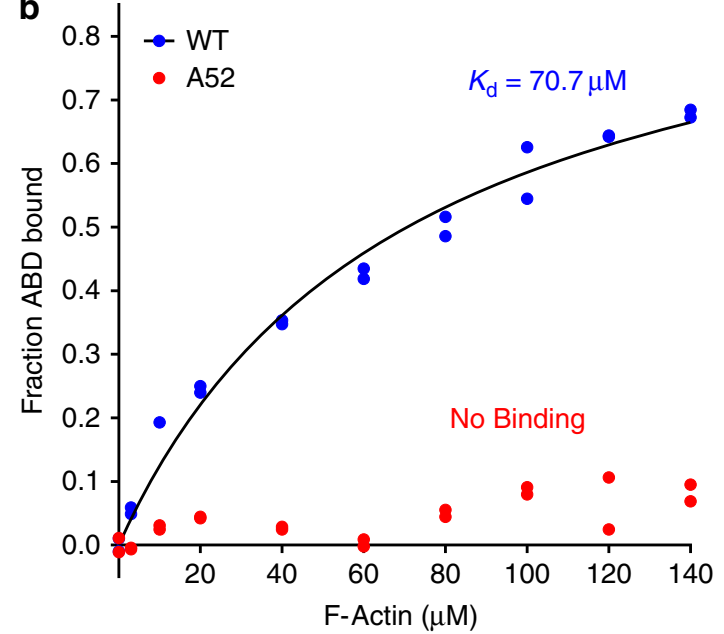

C

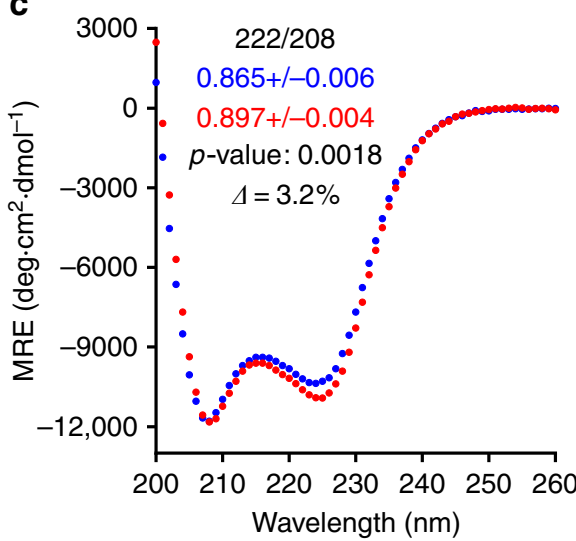

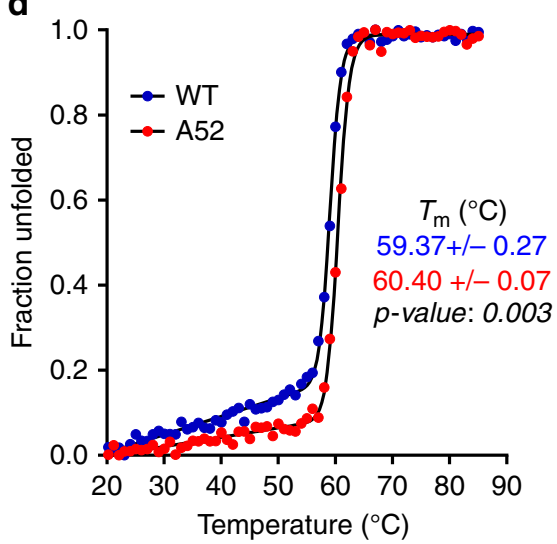

Fig. 2 The $\beta$-III-spectrin $\mathrm{N}$-terminus is required for actin binding. a Coomassie blue stained gel of purified WT ABD or WT ABD without the N-terminal 51 amino acids (A52). $\mathbf{b}$ F-actin co-sedimentation assays showing that the $\mathrm{N}$-terminal truncation abolishes actin affinity. $\mathbf{c}$ CD spectra demonstrating $\alpha$-helical absorption profiles. The A52 ABD has a statistically significant increase in helicity $(n=3)$. d CD denaturation at $222 \mathrm{~nm}$. The A52 ABD has a statistically significant increase in $T_{\mathrm{m}}(n=3)$

Å resolution (Supplementary Fig. 2). This represents a substantial improvement over previous ABD-actin reconstructions ${ }^{13}$, 14, 19, the best of which was $12 \AA$. The reconstruction and resulting atomic model provide several mechanistic insights. First, the density map reveals that only a single $\mathrm{CH}$ domain is bound to actin, as observed previously for the $\alpha$-actinin ABD. Second, the bound $\mathrm{CH}$ domain has an additional $\mathrm{N}$-terminal helix that is tightly associated with actin (Fig. 1b, red). This helix was not identified in other ABD-actin cryo-EM complexes. However, reexamination of the $\alpha$-actinin-actin reconstruction ${ }^{14}$ suggests that extra density is present, consistent with such an N-terminal helical extension. By comparison, the higher resolution fimbrin-actin reconstruction ${ }^{13}$, containing closed $\mathrm{CH}$ domains, shows no extra density. The presence of this contiguous $\mathrm{N}$-terminal helix unambiguously identifies the bound domain as $\mathrm{CH} 1$. Thus, high-affinity actin binding, caused by the L253P mutation in the $\mathrm{CH} 2$ domain, is mediated through the $\mathrm{CH} 1$ domain. The L253P mutation does not expose or generate a de novo high-affinity actin binding site in the $\mathrm{CH} 2$ domain, as has been suggested previously ${ }^{20}$.

All N-terminal ABDs contain amino acid sequences of variable length and composition preceding the conserved $\mathrm{CH} 1$ domain. However, a structured $\mathrm{N}$-terminal region preceding the globular fold of a $\mathrm{CH}$ domain has not been previously observed in most reported ABD crystal structures. This reflects either disorder in this region or the intentional truncation of the region based on predicted intrinsic disorder ${ }^{21}$. However, when calmodulin was crystallized with the plectin $\mathrm{ABD}$, calmodulin was bound to the $\mathrm{N}$-terminal region which had become $\alpha$-helical ${ }^{21}$. Solution studies confirmed that in the absence of calmodulin, the plectin $\mathrm{N}$-terminal region is unstructured. The $\beta$-III-spectrin $\mathrm{CH} 1$ domain with the extended $\mathrm{N}$-terminal helix built into the cryoEM map superimposes very well with the corresponding plectin $\mathrm{CH} 1$ domain with calmodulin (Supplementary Fig. 3), and shows that calmodulin would be involved in massive clashes with actin. As proposed this explains how calmodulin, in the presence of $\mathrm{Ca}^{2}$ ${ }^{+}$, dissociates the plectin $\mathrm{ABD}$ from actin since the binding of actin and calmodulin is competitive ${ }^{22}$.

$\mathrm{N}$-terminal ABD residues are essential for actin binding. The $\beta$-III-spectrin cryo-EM structure showing the $\mathrm{N}$-terminal helix bound to actin suggests that the helix must contribute to binding affinity. To test this, we measured affinity of WT ABD with and without the first 51 amino acids (A52). Strikingly, truncation of the $\mathrm{N}$-terminal sequence abolished binding of the $\mathrm{ABD}$ to actin (Fig. 2a, b). Circular dichroism (CD) indicates that this loss in binding is not due to misfolding (Fig. 2c, d). On the contrary, the A52 ABD showed small but reproducible increases in helicity and stability, suggesting that the $\mathrm{N}$-terminal residues contain intrinsic disorder, which we verified by CD (Supplementary Fig. 4). Collectively, these data sets, combined with the cryo-EM 


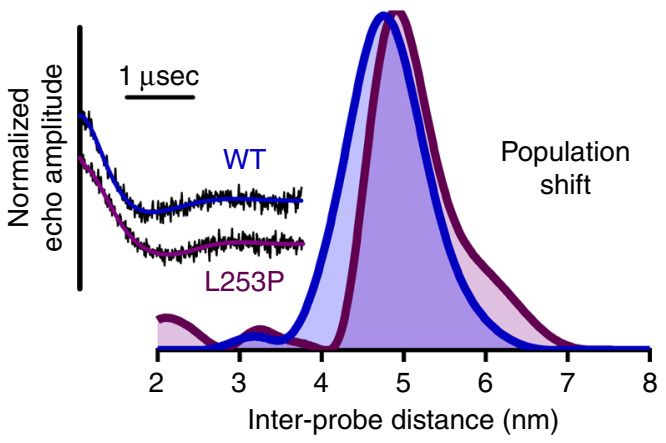

Fig. 3 DEER measurement showing the L253P mutation opens the $\beta$-IIIspectrin $A B D$ structure. Echo amplitude decays of WT ABD (blue) and L253P ABD (purple) along with their corresponding Tikhonov fits are shown on the left. The inter-probe distances derived from Tikhonov regularization (Supplementary Fig. 6) for both WT and L253P ABDs are shown on the right. The WT ABD distance distribution is centered at 4.8 $\mathrm{nm}$, consistent with the distance predicted in the homology model of the closed state shown in Supplementary Fig. 5. Upon introduction of the L253P mutation, the distance distribution undergoes a shift to populate a longer inter-probe distance, visible as a shoulder to the right of the $4.8 \mathrm{~nm}$ peak, consistent with structural opening of the ABD

structural model, indicate that the $\mathrm{N}$-terminal sequence is critical to binding, both directly by interacting with actin and potentially indirectly through allosteric destabilization of the $\mathrm{ABD}$, the latter of which may alter the ABD conformational ensemble to impact binding. Evidence supporting an allosteric contribution comes from recent folding and binding studies on the utrophin ABD1, which showed that the utrophin N-terminal residues destabilize $\mathrm{ABD} 1$ and are required for full $\mathrm{ABD} 1$ binding activity. The utrophin N-terminal residues alone do not bind actin ${ }^{23}$.

L253P mutation promotes structural opening of $\mathrm{CH}$ domains. Previously we demonstrated that the L253P mutation substantially destabilizes the $\operatorname{ABD}\left(\Delta T_{\mathrm{m}}=-14.8^{\circ} \mathrm{C}\right)$. If the open structural state of the $\mathrm{ABD}$ is responsible for high-affinity actin binding, then decreased stability may facilitate opening of the $\mathrm{CH}$ domains from a closed state. In our previous structural homology model of the $\beta$-III-spectrin ABD in the closed structural state ${ }^{7}$, $\mathrm{L} 253$ is positioned at the $\mathrm{CH}$ domain interface (Supplementary Fig. 5), suggesting that the L253P mutation could also perturb $\mathrm{CH} 1-\mathrm{CH} 2$ interactions that stabilize the closed structural state. To test this hypothesis, we used double electron-electron resonance (DEER) to measure inter- $\mathrm{CH}$ domain distance with and without the L253P mutation. We exploited the native cysteine residues at positions 76 and 231 for irreversible attachment of spin labels.

For WT ABD in the absence of actin, clear oscillations were present in the echo amplitude decay (Fig. 3). Analysis (Supplementary Fig. 6) revealed an inter-probe distance centered at $4.8 \mathrm{~nm}$ (Fig. 3), which agrees well with the distance predicted for the closed structural state (Supplementary Fig. 5). Upon introduction of the L253P mutation, the distance distribution undergoes a shift to populate a longer inter-probe distance, visible as a shoulder to the right of the $4.8 \mathrm{~nm}$ peak. This indicates that the $\mathrm{ABD}$ undergoes an opening as a result of the mutation (Fig. 3), consistent with the cryo-EM structure showing that $\mathrm{CH} 1$ separates from $\mathrm{CH} 2$ upon associating with actin (Fig. 1).

In the context of other ABDs harboring disease-causing mutations, a similar structural mechanism has been proposed for the K255E mutation of $\alpha$-actinin- 4 , a missense mutation also located at the $\mathrm{CH}$ domain interface. This mutation caused increased affinity for actin, but the crystal structure showed the
ABD of mutant $\alpha$-actinin- 4 to be in a closed state ${ }^{24}$. While seemingly counter to our proposed mechanism here, the DEERderived distance distributions indicate that the shift to an open conformer is not complete (Fig. 3). Only a small portion of the population exists in the more open state in the absence of actin, with the remaining ensemble occupying the closed conformer. Given that crystallographic conditions favor more stable conformers, the K255E mutant may open, similar to L253P $\beta$-III-spectrin, but crystallize in its more stable closed state because it is more significantly populated.

\section{Discussion}

SCA5 pathology is characterized by atrophy of the cerebellum ${ }^{25}$, likely reflecting degeneration of dendritic arbors extended by Purkinje cells. Within dendrites, $\beta$-III-spectrin binds to actin filaments to form a spectrin-actin skeleton underlying the plasma membrane ${ }^{26}$. The low affinity of WT $\beta$-III-spectrin for actin suggests that normal membrane function requires a dynamic spectrin-actin cytoskeleton in which spectrin-actin linkages form and dissociate. We suggest that the high affinity of L253P $\beta$-IIIspectrin for actin decreases dynamics of spectrin-actin linkages, resulting in reduced plasticity of the spectrin-actin cytoskeleton. We speculate that spectrin-actin cytoskeleton plasticity is important for the cytoskeleton to expand or retract within structurally dynamic regions of the dendritic arbor, such as growing or remodeling dendrites and spines. Recent work has highlighted the requirement of $\beta$-III-spectrin to support dynamic spine structure and post-synaptic signaling ${ }^{6}$. In addition, disrupted microtubule-based transport has been reported for the L253P mutation ${ }^{27}$, and these transport defects may disrupt arborization and contribute to SCA5 pathogenesis. Disrupted transport may be secondary to defects in microtubule tracts that are organized by the spectrin-actin cytoskeleton $28-31$, and/or result from the direct impact of high-affinity binding of L253P $\beta$-III-spectrin to the actin-related protein, ARP $1^{32}$, a component of the dynactin complex that facilitates cargo transport by microtubule motor proteins ${ }^{33}, 34$.

Collectively, the $6.9 \AA$ cryo-EM structure, binding studies, and DEER distance distributions converge on a structural mechanism for disease. The $\mathrm{CH} 2$ domain-localized L253P mutation perturbs a closed-open structural equilibrium in $\beta$-III-spectrin's ABD by lowering the energetic barrier between structural states. The ABD is then relieved of its regulatory mechanism allowing for the $\mathrm{CH} 1$ domain to interact with actin filaments, aided by an additional $\mathrm{N}$ terminal unstructured region that becomes helical upon binding actin.

\section{Methods}

Protein purification. For cryo-EM analyses, the WT or L253P human $\beta$-IIIspectrin ABD coding sequences contained in pET-30a-ABD WT or pET-30a-ABD L253P vectors were expressed in Escherichia coli (E. coli) BL21(DE3) (Novagen). $\mathrm{ABD}$ proteins were both purified using a HiTrap Q $5 \mathrm{~mL}$ ion-exchange column followed by a Superdex 200 size exclusion column (GE Healthcare Life Sciences) ${ }^{7}$. Elution fractions of the Superdex 200 column containing pure ABD proteins as assessed by SDS-PAGE were pooled and concentrated (Amicon Ultra-4 Centrifugal Filter, $10 \mathrm{~K}$ MWCO). A Bradford assay (Biorad) was then used to determine protein concentrations which equaled 44.0 and $40.6 \mu \mathrm{M}$ for WT and L253P, respectively. ABD proteins were stored on ice until preparation of $\mathrm{ABD}-\mathrm{F}$-actin complexes.

To test the contribution of the N-terminus to actin-binding affinity, the coding sequences for WT ABD (amino acids 1-284) or truncated WT ABD (amino acids 52-284) were PCR amplified using the forward primer AAACACCTGCAAAAAG GTATGAGCAGCACGCTGTCACCC or AAACACCTGCAAAAAGGTGCAGAT GAACGAGAAGCTGTGC and reverse primer AAATCTAGACTACTTCATCTT GGAGAAGTAATGGTAGTAAG. PCR products were digested with AarI and $\mathrm{Xbal}$ restriction enzymes and ligated into the BsaI site of pE-SUMOpro (LifeSensors) containing His and SUMO tags. The final constructs pE-SUMO-ABD WT and pE-SUMO-A52-ABD WT were sequence verified and transformed into $E$. coli BL21 (DE3)pLysS (Agilent). Transformed bacteria were incubated with 
rotation at $27^{\circ} \mathrm{C}$ in flasks containing $1 \mathrm{~L} \mathrm{LB}$ media with $100 \mu \mathrm{g}$ per $\mathrm{mL}$ ampicillin and $50 \mu \mathrm{g}$ per $\mathrm{mL}$ chloramphenicol until an absorbance of 0.5 at $550 \mathrm{~nm}$ was reached. Then flasks were placed in ice for $10 \mathrm{~min}$ before addition of IPTG to 0.5 $\mathrm{mM}$ final. The flasks were then incubated with rotation for $4 \mathrm{~h}$ in a $22^{\circ} \mathrm{C}$ water bath. Bacteria were harvested at $5000 \times g$ and pellets stored at $-20^{\circ} \mathrm{C}$. Bacteria were lysed with lysozyme (Sigma) for $1 \mathrm{~h}$ at $4^{\circ} \mathrm{C}$ in buffer containing $50 \mathrm{mM}$ Tris, $\mathrm{pH}$ $7.5,300 \mathrm{mM} \mathrm{NaCl}$, and $25 \%$ sucrose with protease inhibitors (Complete Protease Inhibitor tablet, EDTA-free, Roche), followed by a freeze-thaw cycle using an isopropanol-dry ice bath. Then $\mathrm{MgCl}_{2}$ to $10 \mathrm{mM}$ final and DNasel (Roche) to 7.5 $\mathrm{U}$ per $\mathrm{mL}$ final were added and lysate incubated for $1 \mathrm{~h}$ at $4{ }^{\circ} \mathrm{C}$. Lysate was clarified at $40,000 \times g$ at $4^{\circ} \mathrm{C}$ for $30 \mathrm{~min}$. Supernatants were collected and passed through a $0.45 \mu \mathrm{m}$ syringe filter before loaded onto a Poly-Prep (Biorad) chromatography column containing $1 \mathrm{~mL} \mathrm{Ni-NTA}$ agarose (Qiagen) equilibrated in buffer containing $50 \mathrm{mM}$ Tris, $\mathrm{pH} 7.5,300 \mathrm{mM} \mathrm{NaCl}$, and $20 \mathrm{mM}$ imidazole. The column was washed with buffer containing $50 \mathrm{mM}$ Tris, $\mathrm{pH} 7.5,300 \mathrm{mM} \mathrm{NaCl}$, and $20 \mathrm{mM}$ imidazole, and proteins eluted in buffer containing $50 \mathrm{mM}$ Tris, pH 7.5, $300 \mathrm{mM}$ $\mathrm{NaCl}$, and $150 \mathrm{mM}$ imidazole. Fractions containing ABD proteins were pooled and loaded into a Slide-a-Lyzer, $10 \mathrm{~K}$ MWCO, dialysis cassette (ThermoScientific), and dialysis performed at $4{ }^{\circ} \mathrm{C}$ in buffer containing $25 \mathrm{mM}$ Tris, pH 7.5, $150 \mathrm{mM} \mathrm{NaCl}$ and $5 \mathrm{mM} \beta$-mercaptoethanol. To cleave off the SUMO tag, Ulp1 SUMO protease was added to dialyzed $\mathrm{ABD}$ proteins at a 1:14 (protease:ABD) mass ratio, and digests incubated for $1.5 \mathrm{~h}$ at $4{ }^{\circ} \mathrm{C}$. To separate ABD proteins from the cleaved HisSUMO tag and His-tagged SUMO protease, ABD proteins were loaded onto a Poly-Prep chromatography column containing $0.5 \mathrm{~mL} \mathrm{Ni}-\mathrm{NTA}$ agarose equilibrated in $25 \mathrm{mM}$ Tris, $\mathrm{pH} 7.5,150 \mathrm{mM} \mathrm{NaCl}$, and $5 \mathrm{mM} \beta$-mercaptoethanol. Elution fractions containing ABD proteins were collected and then loaded onto a gel filtration column (Sephadex S100, GE) equilibrated in buffer containing $10 \mathrm{mM}$ Tris, $\mathrm{pH} 7.5,150 \mathrm{mM} \mathrm{NaCl}, 2 \mathrm{mM} \mathrm{MgCl}$, and $1 \mathrm{mM}$ DTT at $4{ }^{\circ} \mathrm{C}$. Fractions were analyzed by SDS-PAGE and Coomassie blue staining, and fractions enriched with $\mathrm{ABD}$ proteins were pooled and concentrated (Amicon Ultra-15 Centrifugal Filter, $10 \mathrm{~K}$ MWCO).

For DEER analyses, the WT and L253P ABD proteins were modified to substitute a serine residue in place of cysteine 115. Spin labeling was performed using native cysteines of which the $\beta$-III-spectrin ABD contains four $(\mathrm{C} 76, \mathrm{C} 115$, C186, C231), and C76, C115, and C231 are all solvent exposed. Residues C76 and C231 were best suited for DEER distance measurements, so C115 was mutated to serine to prevent non-specific labeling at that site. The $\mathrm{C} 186$ site did not require mutagenesis because it is naturally buried in the core of $\mathrm{CH} 2$ and would thus not be accessible to free spin label in solution. PCR site-directed mutagenesis was performed on pET-30a-ABD WT and pET-30a-ABD L253P vector templates using the oligonucleotides CATGCGGATCCACTCCCTGGAGAACGTG and CACGTT CTCCAGGGAGTGGATCCGCATG. The resulting constructs pET-30a-ABD WT C115S and pET-30a-ABD L253P C115S were sequence verified, and then transformed into E. coli BL21(DE3). The ABD C115S proteins were purified as described above. For structural studies of the $\beta$-III-spectrin $\mathrm{N}$-terminal residues, a small peptide corresponding to residues SSTLSPTDFDSLEIQGQYSDINNRWDLP DSDWDNDSSSARLFERSRIKALA was produced via solid-state synthesis through Selleck Chemicals LLC.

Cryo-EM ABD-actin. $5 \mu \mathrm{M}$ of rabbit skeletal muscle G-actin was polymerized in $15 \mathrm{mM}$ Hepes- $\mathrm{HCl}$ buffer, $\mathrm{pH} 7.5,75 \mathrm{mM} \mathrm{KCl}, 1 \mathrm{mM} \mathrm{MgCl}_{2}$, and $0.5 \mathrm{mM}$ ATP for $2 \mathrm{~h}$ at room temperature. For negatively stained samples $2 \mu \mathrm{M} \mathrm{F}$-actin was incubated with 5-10 $\mu \mathrm{M}$ WT $\beta$-III-spectrin or with $2-5 \mu \mathrm{M} \beta$-III-spectrin mutant L253P for 2-20 min. For cryo-samples the mixture $(1.5-2 \mu \mathrm{L})$ was applied to lacey carbon grids. These grids had previously been plasma cleaned (Gatan Solarus), and were then vitrified in a Vitrobot Mark IV (FEI, Inc.). Images were recorded in a Titan Krios operating at $300 \mathrm{keV}$, using a Falcon II camera with $1.05 \AA$ per pixel. The images were dose-fractionated into seven "chunks". Each chunk, a sum of multiple frames, represented a dose of $\sim 20$ electrons $/ \AA^{2}$. A total of 586 images (each $4 \mathrm{k} \times 4 \mathrm{k}$ ) were selected that were free from drift or astigmatism, and had a defocus less than $3.0 \mu \mathrm{m}$. The program CTFFIND $3^{35}$ was used for estimating the defocus and the range used was from 0.6 to $3.0 \mu \mathrm{m}$. The SPIDER software package $^{36}$ was employed for almost all further steps in the image processing. The contrast transfer function (CTF) was corrected by multiplying each image by the theoretical CTF, both reversing phases where they need to be reversed and improving the signal-to-noise ratio (SNR). This operation is simply a Wiener Filter in the limit of a very SNR. The program e2helixboxer within the EMAN $2^{37}$ suite of programs was used for cutting long filaments from the micrographs. Overlapping boxes, each $384 \mathrm{px}$ long with a $40 \mathrm{px}$ shift between adjacent boxes $(\sim 1.5$ times the axial rise per subunit) were extracted from these long filaments, yielding $\sim 60,000$ segments that were padded to $384 \times 384 \mathrm{px}$. The CTF estimation and particle picking came from the integrated images (containing all seven chunks), while the images used for the initial alignments and reconstruction came from only the first two chunks.

An initial reconstruction using the IHRSR method ${ }^{38}$ showed clear decoration of actin, but the mass density due to $\beta$-III-spectrin was lower than that from the actin. This appeared to arise from incomplete occupation. We therefore used atomic models of pure actin and actin decorated with $\alpha$-actinin ${ }^{39}$ to sort the segments. Only approximately one-third of the segments showed a higher cross-correlation with the decorated filament, and 20,340 segments were used for further processing. The IHRSR cycles converged to a rotation of $-166.9^{\circ}$ and an axial rise of $27.3 \AA$ per subunit. After excluding segments with a large out-of-plane tilt or poor orientation against the reference, 12,443 segments were used in the final reconstruction.

Model building. An actin-spectrin asymmetric unit was segmented from the filament map in Chimera ${ }^{40}$. Model building began by docking cryo-EM structure of actin $(5 \mathrm{JLH})^{41}$ and a predicted model of spectrin $\mathrm{CH} 1$ domain into the experimental density data. This predicted spectrin model was generated by ITASSER $^{42}$ based on a crystal structure of plectin $(1 \mathrm{MB} 8)^{43}$. Then the actin-spectrin complex was rebuilt with RosettaCM protocols ${ }^{44}$. A total of 1500 models were generated, and the 10 best models (selected based on Rosetta's energy function) were combined into one model by manual editing in $\operatorname{Coot}^{45}$ to yield the best overall fit to the density map. A filament model was subsequently generated from this and refined by Phenix real-space refinement ${ }^{46}$. MolProbity ${ }^{47}$ was used to evaluate the quality of the model (Supplementary Table 1). The MolProbity scores for the actin-spectrin filament models compare favorably (99th percentile) with structures of similar resolution.

Although segments were sorted to exclude naked actin, Phenix refinement of the actin-spectrin reconstruction clearly shows that the occupancy by spectrin is not $100 \%$, and the actual occupancy is $\sim 75 \%$. Therefore, the threshold chosen for the filament needed to show the full volume for spectrin shows a somewhat larger and lower resolution actin.

Co-sedimentation assays. Actin was purified from acetone powder derived from the psoas muscle of New Zealand white rabbit (Oryctolagus cuniculus) ${ }^{48}$. Acetone powder was hydrated in $4{ }^{\circ} \mathrm{C}$ water for $30 \mathrm{~min}$ to extract actin. The resultant slurry was passed through a Whatman filter paper and $30 \mathrm{mM} \mathrm{KCl}$ was added to the filtrate to polymerize actin for a period of $1 \mathrm{~h}$ at room temperature. Filamentous actin was then pelleted by 30 min centrifugation at $80,000 \mathrm{rpm}$ in a TLA 100.3 rotor. The actin pellet was resuspended in buffer containing $5 \mathrm{mM}$ Tris, $\mathrm{pH}$ 7.6, 0.5 $\mathrm{mM}$ ATP, $0.2 \mathrm{mM} \mathrm{MgCl}$, and homogenized on ice. A 10 min clarifying spin at $70,000 \mathrm{rpm}$ was then performed to pellet aggregate proteins. The G-actin containing supernatant was then isolated and polymerization was initiated by addition of $2 \mathrm{mM} \mathrm{MgCl}$ with a $30 \mathrm{~min}$ incubation at room temperature. The purified ABD proteins were clarified at $100,000 \times g$ at $4{ }^{\circ} \mathrm{C}$ for 30 min prior to setting up binding assays. A Bradford assay was performed to determine F-actin and clarified $\mathrm{ABD}$ protein concentrations. Binding assays were performed in F-buffer containing 10 mM Tris, pH 7.5, $150 \mathrm{mM} \mathrm{NaCl}, 0.5 \mathrm{mM} \mathrm{ATP}, 2 \mathrm{mM} \mathrm{MgCl}_{2}$, and $1 \mathrm{mM}$ DTT. Binding reactions contained $3 \mu \mathrm{M} A B D$ protein and F-actin ranging from 0 to 140 $\mu \mathrm{M}$ in a total volume of $60 \mu \mathrm{L}$. Binding reactions were incubated at room temperature $\left(23-24^{\circ} \mathrm{C}\right)$ for $30 \mathrm{~min}$ to reach equilibrium, and then F-actin pelleted by centrifugation at $100,000 \times g$ at $25^{\circ} \mathrm{C}$ for $30 \mathrm{~min}$. Unbound $\mathrm{ABD}$ was measured by combining $45 \mu \mathrm{L}$ of binding reaction supernatant with $15 \mu \mathrm{L} 4 \times$ Laemmli sample buffer and performing SDS-PAGE followed by Coomassie blue staining. After destaining, gels were scanned using the $700 \mathrm{~nm}$ channel in an Odyssey Imager (LI$\mathrm{COR}$ Biosciences). The fluorescence intensities of the $\mathrm{ABD}$ protein bands were quantified in Image Studio Lite Ver 5.2 software (LI-COR Biosciences) ${ }^{49}$. Individual ABD band fluorescence intensities were converted to amount ABD protein. This conversion was performed using a standard curve generated by linear regression (Prism 5 software) on ABD Coomassie blue fluorescence intensity values attained from a SDS-PAGE gel loaded with varying amounts of ABD in F-buffer. To determine the dissociation constant $\left(K_{\mathrm{d}}\right)$ value, data were fit by non-linear regression in Prism 5 software to the equation:

$$
Y=X /\left(K_{\mathrm{d}}+X\right)
$$

where $Y$ equals fraction $\mathrm{ABD}$ bound and $X$ equals free $\mathrm{F}$-actin concentration

Circular dichroism. ABD proteins were clarified at $100,000 \times g$ for $20 \mathrm{~min}$ at $4{ }^{\circ} \mathrm{C}$ A Bradford assay was performed to determine $\mathrm{ABD}$ protein concentrations, and $\mathrm{ABD}$ proteins were diluted to $250 \mathrm{ng} / \mu \mathrm{L}$ in buffer containing $10 \mathrm{mM}$ Tris, $\mathrm{pH} 7.5$ $150 \mathrm{mM} \mathrm{NaCl}, 2 \mathrm{mM} \mathrm{MgCl} 2,1 \mathrm{mM}$ DTT. CD spectra were acquired in a Jasco J-815 Spectropolarimeter equipped with a Peltier temperature controller. Immediately before analysis, the instrument was baseline-corrected using $\mathrm{ABD}$ protein buffer. For secondary structure analyses, CD spectra were measured from 200 and $260 \mathrm{~nm}$ at $25^{\circ} \mathrm{C}$. Thermal unfolding of the ABD protein sample was analyzed by recording $\mathrm{CD}$ spectra at $222 \mathrm{~nm}$ over the temperature range of $20-85^{\circ} \mathrm{C} . \mathrm{CD}$ analyses were performed three times for each $\mathrm{ABD}$ protein. Non-linear regression analysis was performed in Prism 5 (GraphPad Software, Inc.) to determine the melting temperature using the following equation for a two-state transition, reported previously ${ }^{50}$

$$
Y=\left(\alpha_{N}+\beta_{N} T\right) /\left\{1+e^{4 T_{\mathrm{m}}\left(T-T_{\mathrm{m}}\right) / T \Delta T}\right\}+\left(\alpha D+\beta_{D} T\right) /\left\{1+e^{4 T_{\mathrm{m}}\left(T_{\mathrm{m}}-T\right) / T \Delta T}\right\},
$$

where $Y$ is the $\mathrm{CD}$ signal at temperature $T, T_{\mathrm{m}}$ is melting temperature, $\Delta T$ is the width of the unfolding transition, $\alpha_{\mathrm{N}}$ and $\alpha_{\mathrm{D}}$ are the intercepts of the native and denatured states, respectively, and $\beta_{\mathrm{N}}$ and $\beta_{\mathrm{D}}$ are the slopes of the native and denatured states, respectively.

For secondary structure analysis of the N-terminal peptide, a lyophilized powder was reconstituted in the same buffer system as that described for the ABD 
proteins. The reconstituted peptide was then diluted to a concentration of $99 \mathrm{ng}$ per $\mu \mathrm{L}$ and subsequently scanned over the same wavelength range described above. In the case of both ABDs and peptide, raw ellipticity was normalized to each sample's respective concentrations according to the following equation:

$$
\operatorname{MRE}=[\theta(\mathrm{MW} / N-1)] /(l c),
$$

where $\theta$ represents the raw ellipticity, MW represents the protein molecular weight, $N$ is the number of amino acids, $l$ is the path length, and $c$ is the concentration in milligrams per milliliter.

Statistical analyses. Unpaired, two-tailed $t$-tests were performed in Prism 5 software to determine whether significant differences existed in ABD protein melting temperatures or 222/208 absorbance ratios determined by $\mathrm{CD}$. The $n$ value was equal to three in all cases.

Spin labeling. In the $\beta$-III-spectrin ABD constructs, $500 \mu \mathrm{M}$ of the spin label 4 maleimido-TEMPO (MSL, 4-maleimido-2,2,6,6-tetramethyl-1-piperidinyloxy; Sigma-Aldrich) was added to $25 \mu \mathrm{M}$ protein and equilibrated on a rocker for $3 \mathrm{~h}$ at $4{ }^{\circ} \mathrm{C}$. Prior to addition of MSL, the protein solution had been run over a Zeba desalting column pre-equilibrated with $10 \mathrm{mM}$ Tris, $\mathrm{pH} 7.5$, and $150 \mathrm{mM} \mathrm{NaCl}$ to remove most of the $1 \mathrm{mM}$ DTT left over from size exclusion. After the spin-label incubation period, the protein was once again subjected to a Zeba desalting column to remove any unreacted spin label. To ensure complete removal, however, the spin-labeled protein was then subjected to three $4 \mathrm{~h}$ rounds of dialysis in $4 \mathrm{~L}$ solutions contain $10 \mathrm{mM}$ Tris, $\mathrm{pH} 7.5,150 \mathrm{mM} \mathrm{NaCl}$, and $1 \mathrm{mM}$ DTT. MSL was ultimately chosen over the more commonly used (1-oxyl-2,2,5,5,-tetramethylpyrroline-3-methyl) methanethiosulfonate (MTSSL) because spin labeling of the $\beta$-III-spectrin ABD constructs was incomplete, requiring inclusion of DTT reducing agent post-labeling to prevent undesired $\mathrm{ABD}$ cross-linking. Incubation of the ABD constructs with spin label for periods longer than $3 \mathrm{~h}$ resulted in significant protein loss due to precipitation. The spin-labeled WT and L253P $\beta$-IIIspectrin $\mathrm{ABD}$ constructs were concentrated down to 230 and $175 \mu \mathrm{M}$, respectively, prior to spin counting and DEER sample preparation.

EPR spectroscopy. To verify labeling, a continuous wave EPR spectrum was acquired with sample temperature of $296 \mathrm{~K}$ on the E500 Bruker EPR spectrometer operating at X-band $(9.5 \mathrm{GHz})$ and equipped with an SHQ cavity. The derivative spectrum was then doubly integrated to determine spin concentration by comparing with the double integral of a $100 \mu \mathrm{M}$ TEMPOL standard (Supplementary Fig. 5b, c). For WT and L253P $\beta$-III-spectrin ABD constructs, spin concentrations were determined to be 98 and $75 \mu \mathrm{M}$, respectively, indicating a labeling efficiency of $\sim 43 \%$ for both. After spin counting, we performed DEER on $\beta$-III-spectrin ABD constructs doubly labeled with MSL. ABD samples were prepared by adding $7 \% \mathrm{v} / \mathrm{v}$ glycerol (as a cryoprotectant), loading samples into quartz capillaries $(1.1 \mathrm{~mm}$ i.d., $1.6 \mathrm{~mm}$ o.d., $15 \mu \mathrm{L}$ sample volume) and subsequently flash freezing samples in liquid nitrogen after which samples were stored at $-80^{\circ} \mathrm{C}$ until use. A Bruker E580 spectrometer operating at Q-band $(34 \mathrm{GHz})$ with an EN5107 resonator was then used to implement a four-pulse DEER protocol with a $\pi / 2$ pulse width of $12 \mathrm{~ns}$ and an electron double resonance (ELDOR) pulse width of $24 \mathrm{~ns}^{51}$. The ELDOR frequency was placed on the pump position which corresponded to the absolute maximum of the nitroxide absorption spectrum. The observe position was placed 24 Gauss higher than the pump position on the field swept absorption spectrum. Experiments were run at a temperature $65 \mathrm{~K}$. After data acquisition, backgroundcorrected DEER decays were analyzed using the Tikhonov regularization method provided in DeerAnalysis2013.2 to extract distance distributions encoded in the waveform (Supplemental Fig. 6) ${ }^{52}$. To determine the stable components in the resulting Tikhonov distributions, we examined the impact of a range background models whose starting evolution time varied from 0.5 to $2.4 \mu \mathrm{s}$. The components that were invariable were used for structural interpretation of the ABD constructs. The component that was not stable (the peak at $>7.0 \mathrm{~nm}$ ) was excluded from structural interpretation as it represents an artifact of background subtraction.

Data availability. The reconstruction was deposited in the Electron Microscopy Data Bank with accession number 8886 and the corresponding filament model was deposited in the Protein Data Bank with accession number 6ANU. Other data are available from the corresponding author upon reasonable request.

Received: 29 March 2017 Accepted: 8 September 2017

Published online: 07 November 2017

\section{References}

1. Dick, K. A., Ikeda, Y., Day, J. W. \& Ranum, L. P. Spinocerebellar ataxia type 5. Handb. Clin. Neurol. 103, 451-459 (2012).

2. Ikeda, Y. et al. Spectrin mutations cause spinocerebellar ataxia type 5. Nat. Genet. 38, 184-190 (2006).
3. Stankewich, M. C. et al. Targeted deletion of betaIII spectrin impairs synaptogenesis and generates ataxic and seizure phenotypes. Proc. Natl. Acad. Sci. USA 107, 6022-6027 (2010).

4. Gao, Y. et al. Beta-III spectrin is critical for development of Purkinje cell dendritic tree and spine morphogenesis. J. Neurosci. 31, 16581-16590 (2011).

5. Perkins, E. M. et al. Loss of beta-III spectrin leads to Purkinje cell dysfunction recapitulating the behavior and neuropathology of spinocerebellar ataxia type 5 in humans. J. Neurosci. 30, 4857-4867 (2010).

6. Efimova, N. et al. BetaIII spectrin is necessary for formation of the constricted neck of dendritic spines and regulation of synaptic activity in neurons. $J$. Neurosci. 37, 6442-6459 (2017).

7. Avery, A. W., Crain, J., Thomas, D. D. \& Hays, T. S. A human beta-III-spectrin spinocerebellar ataxia type 5 mutation causes high-affinity F-actin binding. Sci. Rep. 6, 21375 (2016).

8. Banuelos, S., Saraste, M. \& Djinovic Carugo, K. Structural comparisons of calponin homology domains: implications for actin binding. Structure 6 , 1419-1431 (1998).

9. Norwood, F. L., Sutherland-Smith, A. J., Keep, N. H. \& Kendrick-Jones, J. The structure of the N-terminal actin-binding domain of human dystrophin and how mutations in this domain may cause Duchenne or Becker muscular dystrophy. Structure 8, 481-491 (2000).

10. Borrego-Diaz, E. et al. Crystal structure of the actin-binding domain of alphaactinin 1: evaluating two competing actin-binding models. J. Struct. Biol. 155 , 230-238 (2006).

11. Keep, N. H. et al. Crystal structure of the actin-binding region of utrophin reveals a head-to-tail dimer. Structure 7, 1539-1546 (1999).

12. Franzot, G., Sjoblom, B., Gautel, M. \& Djinovic Carugo, K. The crystal structure of the actin binding domain from alpha-actinin in its closed conformation: structural insight into phospholipid regulation of alpha-actinin. J. Mol. Biol. 348, 151-165 (2005).

13. Galkin, V. E., Orlova, A., Cherepanova, O., Lebart, M. C. \& Egelman, E. H. High-resolution cryo-EM structure of the F-actin-fimbrin/plastin ABD2 complex. Proc. Natl. Acad. Sci. USA 105, 1494-1498 (2008).

14. Galkin, V. E., Orlova, A., Salmazo, A., Djinovic-Carugo, K. \& Egelman, E. H. Opening of tandem calponin homology domains regulates their affinity for F-actin. Nat. Struct. Mol. Biol. 17, 614-616 (2010).

15. Orlova, A. A., Galkin, V. E., Nakamura, F. \& Egelman, E. H. Filamin $\mathrm{CH}$ domains bind to F-actin in an open conformation. Mol. Biol. Cell 22, 794a (2011).

16. Way, M., Pope, B. \& Weeds, A. G. Evidence for functional homology in the F-actin binding domains of gelsolin and alpha-actinin: implications for the requirements of severing and capping. J. Cell Biol. 119, 835-842 (1992).

17. Weins, A. et al. Disease-associated mutant alpha-actinin-4 reveals a mechanism for regulating its F-actin-binding affinity. Proc. Natl. Acad. Sci. USA 104, 16080-16085 (2007).

18. Duff, R. M. et al. Mutations in the N-terminal actin-binding domain of filamin C cause a distal myopathy. Am. J. Hum. Genet. 88, 729-740 (2011).

19. Sutherland-Smith, A. J. et al. An atomic model for actin binding by the $\mathrm{CH}$ domains and spectrin-repeat modules of utrophin and dystrophin. J. Mol. Biol. 329, 15-33 (2003).

20. Nakamura, F., Hartwig, J. H., Stossel, T. P. \& Szymanski, P. T. $\mathrm{Ca}^{2+}$ and calmodulin regulate the binding of filamin A to actin filaments. J. Biol. Chem. 280, 32426-32433 (2005).

21. Song, J. G. et al. Structural insights into $\mathrm{Ca}^{2+}$-calmodulin regulation of plectin 1a-integrin beta4 interaction in hemidesmosomes. Structure 23, 558-570 (2015).

22. Kostan, J., Gregor, M., Walko, G. \& Wiche, G. Plectin isoform-dependent regulation of keratin-integrin alpha6beta4 anchorage via $\mathrm{Ca}^{2+} /$ calmodulin. $J$. Biol. Chem. 284, 18525-18536 (2009).

23. Singh, S. M., Bandi, S. \& Mallela, K. M. G. The N-terminal flanking region modulates the actin binding affinity of the utrophin tandem calponinhomology domain. Biochemistry 56, 2627-2636 (2017).

24. Lee, S. H., Weins, A., Hayes, D. B., Pollak, M. R. \& Dominguez, R. Crystal structure of the actin-binding domain of alpha-actinin-4 Lys255Glu mutan implicated in focal segmental glomerulosclerosis. J. Mol. Biol. 376, 317-324 (2008).

25. Bürk, K. et al. Spinocerebellar ataxia type 5: clinical and molecular genetic features of a German kindred. Neurology 62, 327-329 (2004).

26. Han, B., Zhou, R., Xia, C. \& Zhuang, X. Structural organization of the actinspectrin-based membrane skeleton in dendrites and soma of neurons. Proc. Natl. Acad. Sci. USA 114, E6678-E6685 (2017).

27. Lorenzo, D. N. et al. Spectrin mutations that cause spinocerebellar ataxia type 5 impair axonal transport and induce neurodegeneration in Drosophila. J. Cell Biol. 189, 143-158 (2010).

28. Pielage, J. et al. A presynaptic giant ankyrin stabilizes the NMJ through regulation of presynaptic microtubules and transsynaptic cell adhesion. Neuron 58, 195-209 (2008)

29. Koch, I. et al. Drosophila ankyrin 2 is required for synaptic stability. Neuron $\mathbf{5 8}$, 210-222 (2008). 
30. Stephan, R. et al. Hierarchical microtubule organization controls axon caliber and transport and determines synaptic structure and stability. Dev. Cell 33, 5-21 (2015)

31. Eaton, B. A., Fetter, R. D. \& Davis, G. W. Dynactin is necessary for synapse stabilization. Neuron 34, 729-741 (2002).

32. Holleran, E. A. et al. Beta III spectrin binds to the Arp1 subunit of dynactin. J. Biol. Chem. 276, 36598-36605 (2001)

33. Lees-Miller, J. P., Helfman, D. M. \& Schroer, T. A. A vertebrate actin-related protein is a component of a multisubunit complex involved in microtubulebased vesicle motility. Nature 359, 244-246 (1992).

34. Schlager, M. A., Hoang, H. T., Urnavicius, L., Bullock, S. L. \& Carter, A. P. In vitro reconstitution of a highly processive recombinant human dynein complex. EMBO J. 33, 1855-1868 (2014).

35. Mindell, J. A. \& Grigorieff, N. Accurate determination of local defocus and specimen tilt in electron microscopy. J. Struct. Biol. 142, 334-347 (2003).

36. Frank, J. et al. SPIDER and WEB: processing and visualization of images in 3D electron microscopy and related fields. J. Struct. Biol. 116, 190-199 (1996).

37. Tang, G. et al. EMAN2: an extensible image processing suite for electron microscopy. J. Struct. Biol. 157, 38-46 (2007).

38. Egelman, E. H. A robust algorithm for the reconstruction of helical filaments using single-particle methods. Ultramicroscopy 85, 225-234 (2000).

39. Galkin, V. E., Orlova, A., Salmazo, A., Djinovic-Carugo, K. \& Egelman, E. H. Opening of tandem calponin homology domains regulates their affinity for F-actin. Nat. Struct. Mol. Biol. 17, 614-616 (2010).

40. Pettersen, E. F. et al. UCSF Chimera-a visualization system for exploratory research and analysis. J. Comput. Chem. 25, 1605-1612 (2004).

41. von der Ecken, J., Heissler, S. M., Pathan-Chhatbar, S., Manstein, D. J. \& Raunser, S. Cryo-EM structure of a human cytoplasmic actomyosin complex at near-atomic resolution. Nature 534, 724-728 (2016).

42. Zhang, Y. I-TASSER server for protein 3D structure prediction. BMC Bioinformatics 9, 40 (2008).

43. Garcia-Alvarez, B., Bobkov, A., Sonnenberg, A. \& de Pereda, J. M. Structural and functional analysis of the actin binding domain of plectin suggests alternative mechanisms for binding to F-actin and integrin beta4. Structure 11, 615-625 (2003)

44. Wang, R. Y. et al. De novo protein structure determination from near-atomicresolution cryo-EM maps. Nat. Methods 12, 335-338 (2015)

45. Emsley, P. \& Cowtan, K. Coot: model-building tools for molecular graphics. Acta Crystallogr. D Biol. Crystallogr. 60, 2126-2132 (2004).

46. Adams, P. D. et al. PHENIX: a comprehensive Python-based system for macromolecular structure solution. Acta Crystallogr. D Biol. Crystallogr. 66, (213-221 (2010)

47. Chen, V. B. et al. MolProbity: all-atom structure validation for macromolecular crystallography. Acta Crystallogr. D Biol. Crystallogr. 66, 12-21 (2010).

48. Prochniewicz, E., Zhang, Q., Howard, E. C. \& Thomas, D. D. Microsecond rotational dynamics of actin: spectroscopic detection and theoretical simulation. J. Mol. Biol. 255, 446-457 (1996).

49. Butt, R. H. \& Coorssen, J. R. Coomassie blue as a near-infrared fluorescent stain: a systematic comparison with Sypro Ruby for in-gel protein detection. Mol. Cell. Proteomics 12, 3834-3850 (2013).

50. Legardinier, S. et al. A two-amino acid mutation encountered in duchenne muscular dystrophy decreases stability of the rod domain 23 (R23) spectrin-like repeat of dystrophin. J. Biol. Chem. 284, 8822-8832 (2009).
51. Jeschke, G. DEER distance measurements on proteins. Annu. Rev. Phys. Chem 63, 419-446 (2012).

52. Jeschke, G., Koch, A., Jonas, U. \& Godt, A. Direct conversion of EPR dipolar time evolution data to distance distributions. J. Magn. Reson. 155, 72-82 (2002).

\section{Acknowledgements}

This work was partially supported by grants from NIH to T.S.H. (GM44757), D.D.T (AG32961), and E.H.E. (GM81303), and from NSF (MCB-1616854 to D.D.T.). A.W.A. was supported by an NIH grant (GM44757) to T.S.H. and an NIH training grant to D.D.T. (T32 AR007612). M.E.F. was supported by an NIH training grant to D.D.T. (T32 AR007612). The cryo-EM work was conducted at the Molecular Electron Microscopy Core facility at the University of Virginia, which is supported by the School of Medicine and built with NIH grant G20-RR31199. The Titan Krios and Falcon II direct electron detector within the Core were purchased with NIH SIG S10-RR025067 and S10OD018149, respectively.

\section{Author contributions}

A.W.A. and T.S.H. initiated the project. A.W.A. and M.E.F. in consultation with D.D.T and T.S.H. designed the experiments, excluding the cryo-EM and structural modeling that were conducted by F.W., A.O., and E.H.E. A.W.A. and M.E.F. expressed, purified, and characterized all protein samples. A.W.A., M.E.F., A.R.T, and D.D.T. analyzed DEER data. The manuscript was prepared by A.W.A. and M.E.F. with edits by D.D.T., E.H.E., and T.S.H.

\section{Additional information}

Supplementary Information accompanies this paper at doi:10.1038/s41467-017-01367-w.

Competing interests: The authors declare no competing financial interests.

Reprints and permission information is available online at http://npg.nature.com/ reprintsandpermissions/

Publisher's note: Springer Nature remains neutral with regard to jurisdictional claims in published maps and institutional affiliations.

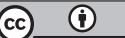

Open Access This article is licensed under a Creative Commons Attribution 4.0 International License, which permits use, sharing, adaptation, distribution and reproduction in any medium or format, as long as you give appropriate credit to the original author(s) and the source, provide a link to the Creative Commons license, and indicate if changes were made. The images or other third party material in this article are included in the article's Creative Commons license, unless indicated otherwise in a credit line to the material. If material is not included in the article's Creative Commons license and your intended use is not permitted by statutory regulation or exceeds the permitted use, you will need to obtain permission directly from the copyright holder. To view a copy of this license, visit http://creativecommons.org/ licenses/by/4.0/.

(ㄷ) The Author(s) 2017 